\title{
Topic: A study on the association of conflict management competencies with emotional intelligence of software professionals in IT industry in India.
}

\author{
${ }^{1}$ Sashakt Singh, ${ }^{2}$ Prof. IleyasRizvi, \\ ${ }^{1}$ Research scholar, Kalinga University.Managing Director, Aryakul Group of colleges. \\ ${ }^{2}$ Director, Management \& Research Institute.
}

\begin{abstract}
In the present study, aim of the researcher is to investigate the association of conflict management competencies and Emotional Intelligence ofsoftware professionals in IT industry in India. The study was conducted in IT industry in India. A structured questionnaire was developed by researcher based on conflict management competencies and its association with emotional intelligence. A sample of 500 software professionals' primary data was collected through convenience sampling technique and chi square test was used for data analysis which is the best tool to check whether there is association between two variables or not. This analysis is followed by crosstabulation. The statistical data were computed by SPSS 21.0 for Windows. The analysis found statistically significant positive association between independent and dependentvariables of the present study; this means that increased emotional intelligence scores were very clearly associated with increased score of conflict management competencies of software professionals in Indian IT.
\end{abstract}

Keywords:Emotional Intelligence, Conflict Management, Software Professionals, IT industry.

\section{Introduction}

The IT industry is witnessing rapid change and speedy growth. The change in technology is lessening the life of many IT products even within a span of a year, and the prices of such products and technology continue to fall. Because of the cut throat competition and various environmental pressures faced by the IT industry, more sophisticated skilled, highly empowered and more creative software professional are needed to serve more demanding clients or customers. These factors have a significant role in increasing the effectiveness and efficiency of software professionals in IT industry in India. To deal with these challenges employees need not only technical skills but other skills commonly known as emotional skills, as explained by Goleman (1999) that the personal and social competencies in Emotional Intelligence (EI) enhance an individual's high technical and functional expertise and for software engineers EI means a balance of technical and emotional considerations to problem solving scenarios.

\subsection{Research Questions Guiding the Study}

The key question this study addresses is whether conflict management competencies are associated with emotional intelligence or not.

- What is association between conflict management competencies emotional intelligence software professional in India?

- What is the relationship among the factors that determine conflict management competencies and emotional intelligence of software professionals in I.T. Industry?

The purpose of this research will be to draw conclusions about the relationship of factors of conflict management competencies and software professionals' emotional intelligence in I.T. industry.

\subsection{Purpose\& Aim of the Research}

Conceivably, a software professional is also a manager with the ability to perceive, understand, manage, and use emotions to serve clients and can deliver IT services. Furthermore, the awareness and refinement of emotional intelligence will be an investment in human resources in the organization. Emotionally intelligent software professional leadership may lessen personnel turnover and improve efficiency among other software professionals and most importantly the clients.

\subsection{Research objective}

Research objectives: To investigate the association between the various variables of conflict management competencies withEmotional Intelligence of Software professionals in I.T. industry. 


\section{Literature Review}

\subsection{Emotional Intelligence in I.T. Industry}

The relationship between clients and software professional is an emotion-laden experience for both participants. Emotional intelligence has an obvious role in I.T. services on both sides. This research will examine the impact of emotional intelligence from both the clients' and software professionals' perspectives. On the clients' side, emotional intelligence may have a role in urgent need and in the usual requirement. Forsoftware professionals, clients' satisfaction may be improved by enhancing and valuing their emotional intelligence and conflict management competencies.Emotionally intelligent software professionals are believed to be a need of hour and an essential part in creating an effective \& successful organisational performance. Some researchers have begun to explore the role of EI and client-centered approach and while research has yet to conclusively weigh in on the subject, common sense would suggest that providers with the high EI would be more likely to connect and communicate successfully with clients (Birks \& Watt, 2007).Over time, researchers have attempted to unite emotion with intelligence though term "emotional intelligence" was not always used, but it was expressed in different ways. This concept has been studied and considered since the beginning of the twentieth century (Goleman, 1995), and Edward Thorndike (1920), researched upon the dimensions of EI as a form of "social intelligence". He investigated the social intelligence as a major component of intelligence measured through the IQ score.

Gardner (1983), wrote about "multiple intelligences" and he further divided it into "intrapersonal" and "interpersonal" intelligences. Later on these intelligences became the foundation for the present models of EI (Goleman, 1995).

However, the term 'Emotional Intelligence' (EI) was used by Peter Salovey and John Mayer in their article, "Emotional Intelligence" in research journal named Imagination, Cognition, and Personality in year 1990 and they presented the first model of emotional intelligence. Denial Goleman (1995) made popular this term by his book entitled Emotional Intelligence: Why It Can Matter More Than IQ (1995).

EI was describes as "abilities such as being able to motivate oneself and persist in the face of frustrations; to control impulse and delay gratification; to regulate one's moods and keep distress from swamping the ability to think; to empathize and to hope."

Golemandivided emotional competence into two categories.

- Personal Competences: which determines how individual manages himself, this includes self-awareness, self-regulation and self-motivation.

- Social Competences: this looks at how one manages his relationships and includes empathy and social skills with a purpose (Goleman, 1998).

\subsection{The Components of Emotional Intelligence}

Shapiro (2002),pointed out in his research that there several components of emotional intelligence which are :

- Moral feeling

- Intellectual skills

- Problems solving

- Social skills

- Achievement skills

- Self-motivation

- The power of empathize

Salovey, Mayer, Turvey, and Palfai (1995) proposed a model of emotional intelligence that includes abilities in five domains:

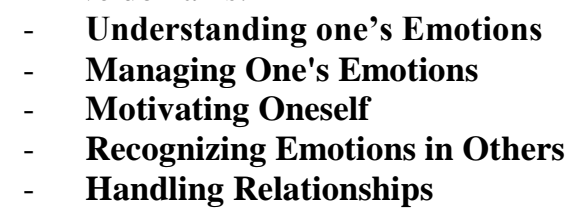

A definition of intelligence that provides a useful theoretical framework for considering the EI construct was recently proposed by Sternberg (1997): Intelligence comprises the mental abilities necessary for adaptation to, as well as shaping and selection of, any environmental contents. According to this definition, individuals act intelligently not only when they successfully adapt or react to the environment, but also when they shape and change their existing environment to meet their needs. Sternberg saidthat intelligence has a common core of mental processes, irrespective of culture or environmental context.

Daniel Goleman (2001)have covered in their book 'The Emotionally Intelligent Workplace' that how organizations can increase emotional intelligence through use of standard human resource functions, such as hiring and performance management systems. 
A study conducted by Carmeli (2003) demonstrated following results:

- Senior managers having high emotional intelligence tend to develop high affective commitment to the organization \& career,

- Positive and significant relationship between emotional intelligence and job satisfaction,

- Managers having high EI are more likely to control work-family conflict and

- Managers having high EI perform better than managers having low EI.

\section{Research Design And Methodology}

A research design is a plan or blueprint of how the researcher is set to conduct the research. The research design basically focuses on the final results: What kind of study is being planned and what kind of result is aimed at? Research methodology focuses on the research process and the kind of statistical tools and procedures are to be used.

\subsection{Methodology}

In order to study the emotional intelligence of software professionals of I.T. industry, an Emotional Intelligence test in form of questionnaire / schedulewas used which was based on sevencomponents namely: personal competence, social competence, optimism, pessimism, self-awareness, empathy and resilience. Structured questionnaire, having multiple choices, dichotomous, objective questions (5 point LIKERT scale) was used. The Sample Unitwas a software professional working in the I.T. industry in India and the Sample Size for the proposed research will be 500 which was taken from all over India, the Universe of the research to know the emotional intelligence of the software professionals. The researcher usedConvenient Sampling Technique to collect primary data with the help of Questionnaires as well as Schedules. Questionnaires were sent to the software professionals through emails also.

\subsection{Research Hypotheses}

Hypotheses are statements that describe the relationship or difference between two or more variables related to the research problem or statement. Following research objectives are formed to address the research problems and test the research hypotheses-

Alternate Hypothesis(H1): There is a positive/significant association between the various variables of conflict management competencies with Emotional Intelligence of Software professionals in I.T. industry.

Null Hypothesis(H0): There is no positive/significant association between the various variables of conflict management competencies with Emotional Intelligence of Software professionals in I.T. industry.

\section{Data Analysis \& Interpretationand Findings}

\subsection{Demographic Profile of the Software Professionals working in IT Industry in India}

Table 4.1 Age

\begin{tabular}{|l|l|l|l|l|l|}
\hline \multicolumn{2}{|l|}{ AGE } & Frequency & Percent & Valid Percent & Cumulative Percent \\
\hline \multirow{5}{*}{ Valid } & $20-30$ & 321 & 64.2 & 64.2 & 64.2 \\
\cline { 2 - 7 } & $31-40$ & 156 & 31.2 & 31.2 & 95.4 \\
\cline { 2 - 7 } & $41-50$ & 15 & 3.0 & 3.0 & 98.4 \\
\cline { 2 - 7 } & ABOVE 50 & 8 & 1.6 & 1.6 & 100.0 \\
\cline { 2 - 7 } & Total & 500 & 100.0 & 100.0 & \\
\hline
\end{tabular}

Table 4.2 Gender

\begin{tabular}{|l|l|l|l|l|l|}
\hline GENDER & Frequency & Percent & Valid Percent & Cumulative Percent \\
\hline \multirow{3}{*}{ Valid } & MALE & 353 & 70.6 & 70.6 & 70.6 \\
\cline { 2 - 7 } & FEMALE & 147 & 29.4 & 29.4 & 100.0 \\
\cline { 2 - 6 } & Total & 500 & 100.0 & 100.0 & \\
\hline
\end{tabular}

Table 4.3 Marital Status

\begin{tabular}{|c|c|c|c|c|c|}
\hline \multicolumn{6}{|c|}{ MARITAL STATUS } \\
\hline & & Frequency & Percent & Valid Percent & Cumulative Percent \\
\hline \multirow{3}{*}{ Valid } & SINGLE & 166 & 33.2 & 33.2 & 33.2 \\
\hline & MARRIED & 334 & 66.8 & 66.8 & 100.0 \\
\hline & Total & 500 & 100.0 & 100.0 & \\
\hline
\end{tabular}

Table 4.4 Educational Qualifications

\begin{tabular}{|c|c|c|c|c|c|}
\hline \multicolumn{6}{|c|}{ Educational Qualifications } \\
\hline & & Frequency & Percent & Valid Percent & Cumulative Percent \\
\hline \multirow{6}{*}{ Valid } & Diploma Holder & 149 & 29.8 & 29.8 & 29.8 \\
\hline & MCA/M.Tech & 114 & 22.8 & 22.8 & 52.6 \\
\hline & B.TECH & 112 & 22.4 & 22.4 & 75.0 \\
\hline & OTHERS(Graduation / Post Graduation) & 16 & 3.2 & 3.2 & 78.2 \\
\hline & Other Technical Qualification & 109 & 21.8 & 21.8 & 100.0 \\
\hline & Total & 500 & 100.0 & 100.0 & \\
\hline
\end{tabular}


Table 4.5 Designation

\begin{tabular}{|c|c|c|c|c|c|}
\hline \multicolumn{6}{|c|}{ Designation } \\
\hline & & Frequency & Percent & Valid Percent & Cumulative Percent \\
\hline \multirow{4}{*}{ Valid } & Junior level & 205 & 41.0 & 41.0 & 41.0 \\
\hline & Middle level & 254 & 50.8 & 50.8 & 91.8 \\
\hline & Senior level & 41 & 8.2 & 8.2 & 100.0 \\
\hline & Total & 500 & 100.0 & 100.0 & \\
\hline
\end{tabular}

\section{Interpretation \& Findings}

The main demographic information of respondents is summarized below:

In case of the software professionals in IT industry in India, the majority of thesoftware professionals surveyedwere males $(70.6 \%)$ and females were $(29.4 \%)$. We can see that majority $64.2 \%$ software professionals belong to 20-30 age group; And 66.8\% respondents were married and 33.2\% respondents were unmarried.

$29.8 \%$ respondents were diploma holders, $22.8 \%$ respondents were MCA / M.Tech, $22.4 \%$ respondents were B.Tech. and $21.8 \%$ respondents have other technical qualification.Designation of $41.0 \%$ respondents was junior level, designation of $50.8 \%$ respondents was middle level \& designation of $8.2 \%$ respondents was senior level

4.2. Analysis: Analysis of the relationship between emotional intelligence and Conflict Management Competencies of the software professional in India.

4.2.1. Analysis: Analysis of the relationship between emotional intelligence andI bring disagreements into the open in order to de-escalate it of the software professional in India.

$\mathbf{H}_{0}$ : The two factors are independent.

$\mathbf{H}_{1}$ : The two factors are not independent (associated).

Tool Used: Chi Sourare Test (Analvze $\rightarrow$ Descrintive Statistics $\rightarrow$ Crosstabs)

Table 4.6Chi-Square Tests

\begin{tabular}{|c|c|c|c|}
\hline \multicolumn{4}{|l|}{ Chi-Square Tests } \\
\hline & Value & $\mathrm{df}$ & Asymp. Sig. (2-sided) \\
\hline Pearson Chi-Square & $58.308^{\mathrm{a}}$ & 16 & .000 \\
\hline Likelihood Ratio & 55.945 & 16 & .000 \\
\hline Linear-by-Linear Association & 2.530 & 1 & .112 \\
\hline $\mathrm{N}$ of Valid Cases & 500 & & \\
\hline
\end{tabular}

Table-4.7. Symmetric Measures:

\begin{tabular}{|l|l|l|l|}
\hline \multicolumn{2}{|l|}{ Symmetric Measures } & Value & Approx. Sig. \\
\hline & Contingency Coefficient & .323 & .000 \\
\hline Nominal by Nominal & 500 & \\
\hline N of Valid Cases & \\
\hline a. Not assuming the null hypothesis. \\
\hline b. Using the asymptotic standard error assuming the null hypothesis. \\
\hline
\end{tabular}

Interpretation: From the table we find out that asymptotic significance for Pearson Chi Square comes out to be 0.000 (less than 0.05 ) so we reject null hypothesis at $5 \%$ level of significance. Hence it can be concluded that two variables are associated.

Table-4.8. Crosstab: I bring disagreements into the open in order to de-escalate it.

\begin{tabular}{|c|c|c|c|c|c|c|c|c|}
\hline \multicolumn{9}{|l|}{ Crosstab } \\
\hline & & & \multicolumn{5}{|c|}{ I bring disagreements into the open in order to de-escalate it. } & \multirow[t]{2}{*}{ Total } \\
\hline & & & $\begin{array}{l}\text { STRONGLY } \\
\text { AGREE }\end{array}$ & AGREE & NEUTRAL & DISAGREE & $\begin{array}{l}\text { STRONGLY } \\
\text { DISAGREE }\end{array}$ & \\
\hline \multirow{10}{*}{$\begin{array}{l}\text { I am emotionally } \\
\text { intelligent. }\end{array}$} & \multirow{2}{*}{$\begin{array}{l}\text { STRONGLY } \\
\text { AGREE }\end{array}$} & Count & 21 & 79 & 26 & 4 & 4 & 134 \\
\hline & & $\%$ of Total & $4.2 \%$ & $15.8 \%$ & $5.2 \%$ & $0.8 \%$ & $0.8 \%$ & $26.8 \%$ \\
\hline & \multirow{2}{*}{ AGREE } & Count & 62 & 69 & 39 & 21 & 7 & 198 \\
\hline & & $\%$ of Total & $12.4 \%$ & $13.8 \%$ & $7.8 \%$ & $4.2 \%$ & $1.4 \%$ & $39.6 \%$ \\
\hline & \multirow{2}{*}{ NEUTRAL } & Count & 45 & 40 & 38 & 13 & 6 & 142 \\
\hline & & $\%$ of Total & $9.0 \%$ & $8.0 \%$ & $7.6 \%$ & $2.6 \%$ & $1.2 \%$ & $28.4 \%$ \\
\hline & \multirow{2}{*}{ DISAGREE } & Count & 3 & 7 & 3 & 7 & 0 & 20 \\
\hline & & $\%$ of Total & $0.6 \%$ & $1.4 \%$ & $0.6 \%$ & $1.4 \%$ & $0.0 \%$ & $4.0 \%$ \\
\hline & \multirow{2}{*}{$\begin{array}{l}\text { STRONGLY } \\
\text { DISAGREE }\end{array}$} & Count & 0 & 3 & 3 & 0 & 0 & 6 \\
\hline & & $\%$ of Total & $0.0 \%$ & $0.6 \%$ & $0.6 \%$ & $0.0 \%$ & $0.0 \%$ & $1.2 \%$ \\
\hline \multirow{2}{*}{\multicolumn{2}{|c|}{ Total }} & Count & 131 & 198 & 109 & 45 & 17 & 500 \\
\hline & & $\%$ of Total & $26.2 \%$ & $39.6 \%$ & $21.8 \%$ & $9.0 \%$ & $3.4 \%$ & $100.0 \%$ \\
\hline
\end{tabular}

Interpretation \& Findings: from the above crosstab, it can said that out of total 500 respondents (Software Professionals), 26.2\% respondents strongly agreed, $39.6 \%$ respondents agreed, $21.8 \%$ respondents were neutral, $9.0 \%$ respondents disagreed and $3.4 \%$ respondents strongly disagreed that 'I bring disagreements into the open in order to de-escalate it. 


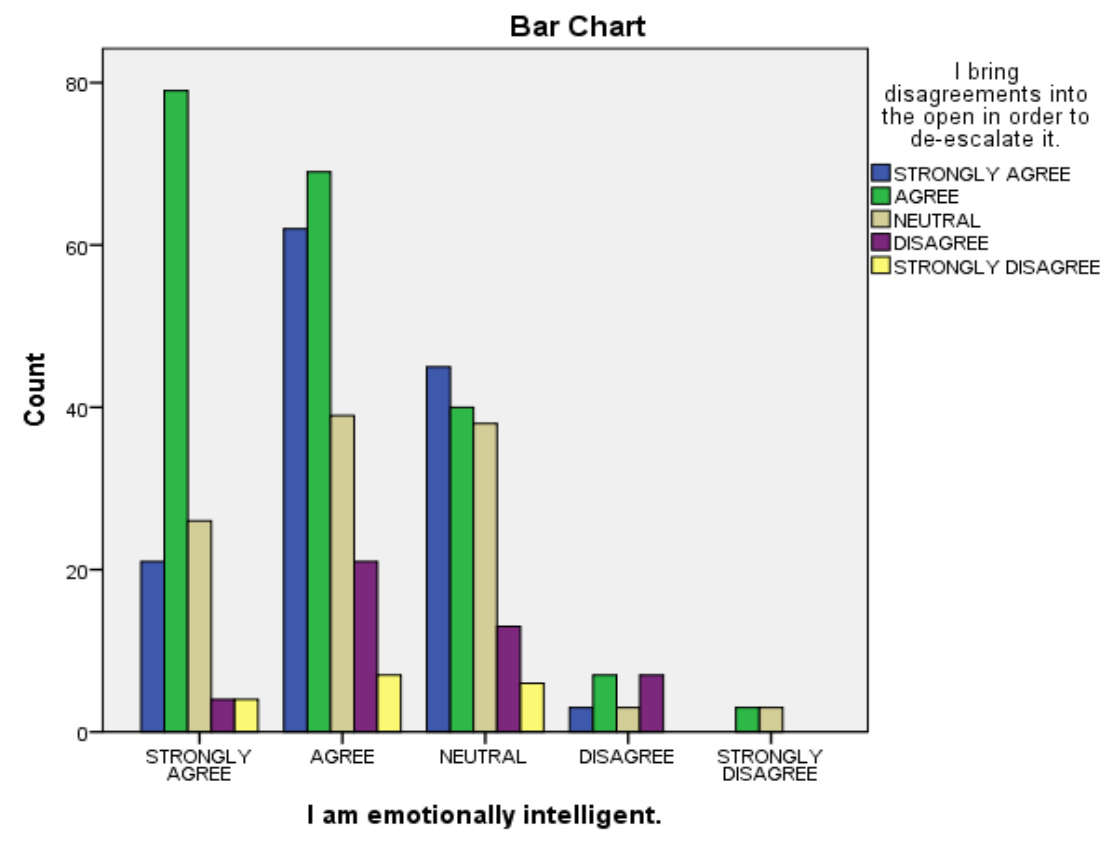

4.2.2. Analysis: Analysis of the relationship between emotional intelligence andI am conscious of my needs

\begin{tabular}{|c|c|c|c|}
\hline \multicolumn{4}{|c|}{$\begin{array}{l}\mathrm{H}_{0}: \text { The two factors are independent. } \\
\mathrm{H}_{1} \text { : The two factors are not independent (associated). } \\
\text { Tool Used: Chi Square Test (Analyze } \rightarrow \text { Descriptive Statistics } \rightarrow \text { Crosstabs) }\end{array}$} \\
\hline \multicolumn{4}{|c|}{ the software professional in India. } \\
\hline \multicolumn{4}{|c|}{ Table 4.9 Chi-Square Tests } \\
\hline \begin{tabular}{|l|} 
Chi-Square Tests \\
\end{tabular} & & & \\
\hline & Value & Df & Asymp. Sig. (2-sided) \\
\hline \begin{tabular}{|l|} 
Pearson Chi-Square \\
\end{tabular} & $82.293^{\mathrm{a}}$ & 16 & .000 \\
\hline Likelihood Ratio & 56.545 & 16 & .000 \\
\hline Linear-by-Linear Association & .331 & 1 & .565 \\
\hline $\mathrm{N}$ of Valid Cases & 500 & & \\
\hline
\end{tabular}

and

want

$s$ in

life

of

Table-4.10. Symmetric Measures:

\begin{tabular}{|l|l|l|l|}
\hline \multicolumn{2}{|l|}{ Symmetric Measures } \\
\hline \multicolumn{2}{|l|}{} & Value & Approx. Sig. \\
\hline Nominal by Nominal & Contingency Coefficient & .376 & .000 \\
\hline N of Valid Cases & 500 & \\
\hline a. Not assuming the null hypothesis. \\
\hline b. Using the asymptotic standard error assuming the null hypothesis. \\
\hline
\end{tabular}

Interpretation \& Findings: From the table we find out that asymptotic significance for Pearson Chi Square comes out to be 0.000 (less than 0.05 ) so we reject null hypothesis at $5 \%$ level of significance. Hence it can be concluded that two variables are associated.

Table-4.11. Crosstab:I carefully arrange win-win solutions.

\begin{tabular}{|c|c|c|c|c|c|c|c|c|}
\hline \multicolumn{9}{|l|}{ Crosstab } \\
\hline & & & \multicolumn{5}{|c|}{ I carefully arrange win-win solutions. } & Total \\
\hline \multirow{4}{*}{$\begin{array}{l}\text { I am emotionally } \\
\text { intelligent. }\end{array}$} & $\begin{array}{l}\text { STRONGLY } \\
\text { AGREE }\end{array}$ & $\%$ of Total & $7.6 \%$ & $7.8 \%$ & $6.8 \%$ & $3.0 \%$ & $1.6 \%$ & $26.8 \%$ \\
\hline & \multirow{2}{*}{ AGREE } & Count & 43 & 88 & 57 & 6 & 4 & 198 \\
\hline & & $\%$ of Total & $8.6 \%$ & $17.6 \%$ & $11.4 \%$ & $1.2 \%$ & $0.8 \%$ & $39.6 \%$ \\
\hline & NEUTRAL & $\%$ of Total & $6.2 \%$ & $10.8 \%$ & $9.4 \%$ & $2.0 \%$ & $0.0 \%$ & $28.4 \%$ \\
\hline
\end{tabular}


Topic: A study on the association of conflict management competencies with emotional intelligence of

\begin{tabular}{|l|l|l|l|l|l|l|l|l|}
\hline \multirow{5}{*}{ DISAGREE } & Count & 7 & 6 & 3 & 4 & 0 & 20 \\
\cline { 3 - 9 } & & $\%$ of Total & $1.4 \%$ & $1.2 \%$ & $0.6 \%$ & $0.8 \%$ & $0.0 \%$ & $4.0 \%$ \\
\cline { 3 - 9 } & STRONGLY & Count & 0 & 0 & 3 & 0 & 3 & 6 \\
\cline { 3 - 9 } & DISAGREE & $\%$ of Total & $0.0 \%$ & $0.0 \%$ & $0.6 \%$ & $0.0 \%$ & $0.6 \%$ & $1.2 \%$ \\
\hline \multirow{2}{*}{ Total } & Count & 119 & 187 & 144 & 35 & 15 & 500 \\
\cline { 3 - 8 } & & $\%$ of Total & $23.8 \%$ & $37.4 \%$ & $28.8 \%$ & $7.0 \%$ & $3.0 \%$ & $100.0 \%$ \\
\hline
\end{tabular}

Interpretation \& Findings: from the above crosstab, it can said that out of total 500 respondents (Software Professionals), 23.8\% respondents strongly agreed, 37.4\% respondents agreed, $28.8 \%$ respondents were neutral, $7.0 \%$ respondents disagreed and $3.0 \%$ respondents strongly disagreed that 'I carefully arrange win-win solutions..'

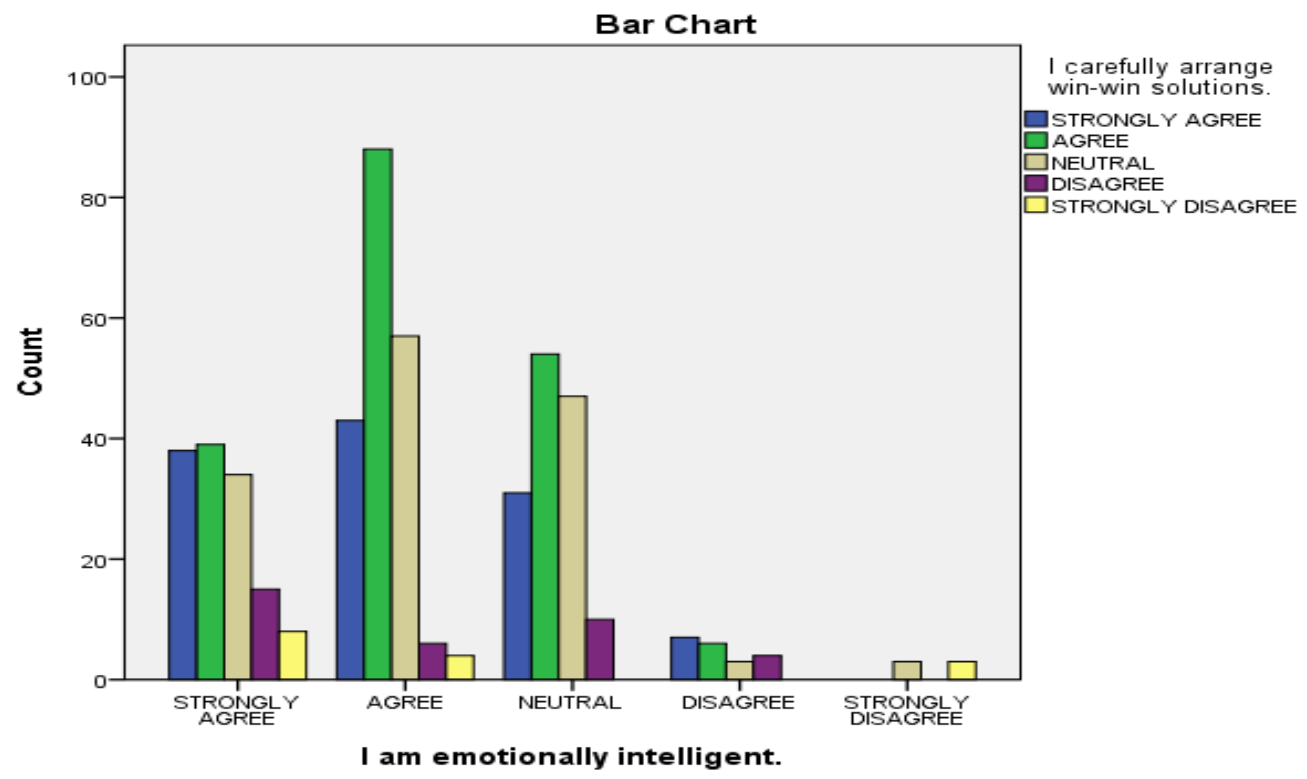

4.2.3.Analysis: Analysis of the relationship between emotional intelligence and'I prefer tackling obstacles and problems rather than simply complaining about them' of the software professional in India.

$\mathrm{H}_{0}$ : The two factors are independent.

$\mathbf{H}_{1}$ : The two factors are not independent (associated).

Tool Used: Chi Square Test (Analyze $\rightarrow$ Descriptive Statistics $\rightarrow$ Crosstabs)

Table 4.12 Chi-Square Tests

\begin{tabular}{|l|l|l|l|}
\hline Chi-Square Tests & Value & Df & Asymp. Sig. (2-sided) \\
\hline & $81.877^{\mathrm{a}}$ & 16 & .000 \\
\hline Pearson Chi-Square & 62.311 & 16 & .000 \\
\hline Likelihood Ratio & 2.676 & 1 & .102 \\
\hline Linear-by-Linear Association & 500 & & \\
\hline N of Valid Cases & \multicolumn{2}{l}{} \\
\hline a. 10 cells (40.0\%) have expected count less than 5. The minimum expected count is .20. & \\
\hline
\end{tabular}

Table-4.13. Symmetric Measures:

\begin{tabular}{|c|c|c|c|}
\hline \multicolumn{4}{|l|}{ Symmetric Measures } \\
\hline & & Value & Approx. Sig \\
\hline Nominal by Nominal & Contingency Coefficient & .375 & .000 \\
\hline \multicolumn{2}{|l|}{$\mathrm{N}$ of Valid Cases } & 500 & \\
\hline
\end{tabular}

Interpretation \& Findings: From the table we find out that asymptotic significance for Pearson Chi Square comes out to be 0.000 (less than 0.05 ) so we reject null hypothesis at $5 \%$ level of significance. Hence it can be concluded that two variables are associated.

Table-4.14. Crosstab: I prefer tackling obstacles and problems rather than simply complaining about them.

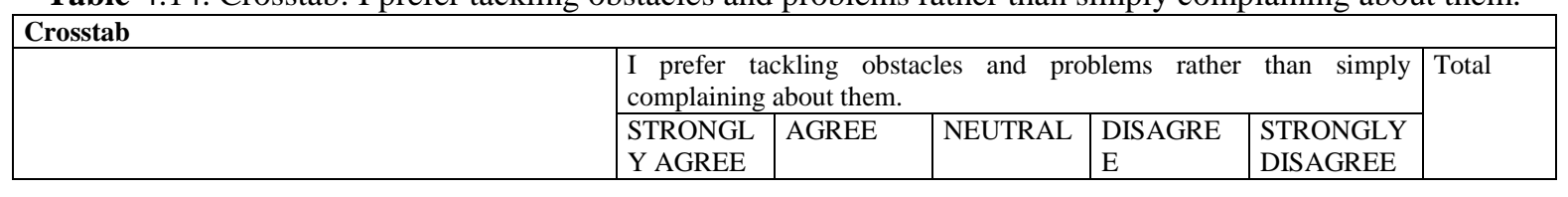


Topic: A study on the association of conflict management competencies with emotional intelligence of

\begin{tabular}{|c|c|c|c|c|c|c|c|c|}
\hline \multirow{10}{*}{$\begin{array}{l}\text { I am emotionally } \\
\text { intelligent. }\end{array}$} & \multirow{2}{*}{$\begin{array}{l}\text { STRONGLY } \\
\text { AGREE }\end{array}$} & Count & 29 & 53 & 33 & 15 & 4 & 134 \\
\hline & & $\%$ of Total & $5.8 \%$ & $10.6 \%$ & $6.6 \%$ & $3.0 \%$ & $0.8 \%$ & $26.8 \%$ \\
\hline & \multirow{2}{*}{ AGREE } & Count & 69 & 71 & 38 & 10 & 10 & 198 \\
\hline & & $\%$ of Total & $13.8 \%$ & $14.2 \%$ & $7.6 \%$ & $2.0 \%$ & $2.0 \%$ & $39.6 \%$ \\
\hline & \multirow{2}{*}{ NEUTRAL } & Count & 38 & 45 & 27 & 32 & \begin{tabular}{|l|}
0 \\
\end{tabular} & 142 \\
\hline & & $\%$ of Total & $7.6 \%$ & $9.0 \%$ & $5.4 \%$ & $6.4 \%$ & $0.0 \%$ & $28.4 \%$ \\
\hline & \multirow{2}{*}{ DISAGREE } & Count & 3 & 7 & 7 & 3 & 0 & 20 \\
\hline & & $\%$ of Total & $0.6 \%$ & $1.4 \%$ & $1.4 \%$ & $0.6 \%$ & $0.0 \%$ & $4.0 \%$ \\
\hline & \multirow{2}{*}{$\begin{array}{l}\text { STRONGLY } \\
\text { DISAGREE }\end{array}$} & Count & 0 & 3 & 0 & 0 & 3 & 6 \\
\hline & & $\%$ of Total & $0.0 \%$ & $0.6 \%$ & $0.0 \%$ & $0.0 \%$ & $0.6 \%$ & $1.2 \%$ \\
\hline \multirow{2}{*}{\multicolumn{2}{|c|}{ Total }} & Count & 139 & 179 & 105 & 60 & 17 & 500 \\
\hline & & $\%$ of Total & $27.8 \%$ & $35.8 \%$ & $21.0 \%$ & $12.0 \%$ & $3.4 \%$ & $100.0 \%$ \\
\hline
\end{tabular}

Interpretation \& Findings: from the above crosstab, it can said that out of total 500 respondents (Software Professionals), $27.8 \%$ respondents strongly agreed, $35.8 \%$ respondents agreed, $21.0 \%$ respondents were neutral, $12.0 \%$ respondents disagreed and $3.4 \%$ respondents strongly disagreed that ' $\mathbf{I}$ prefer tackling obstacles and problems rather than simply complaining about them.'

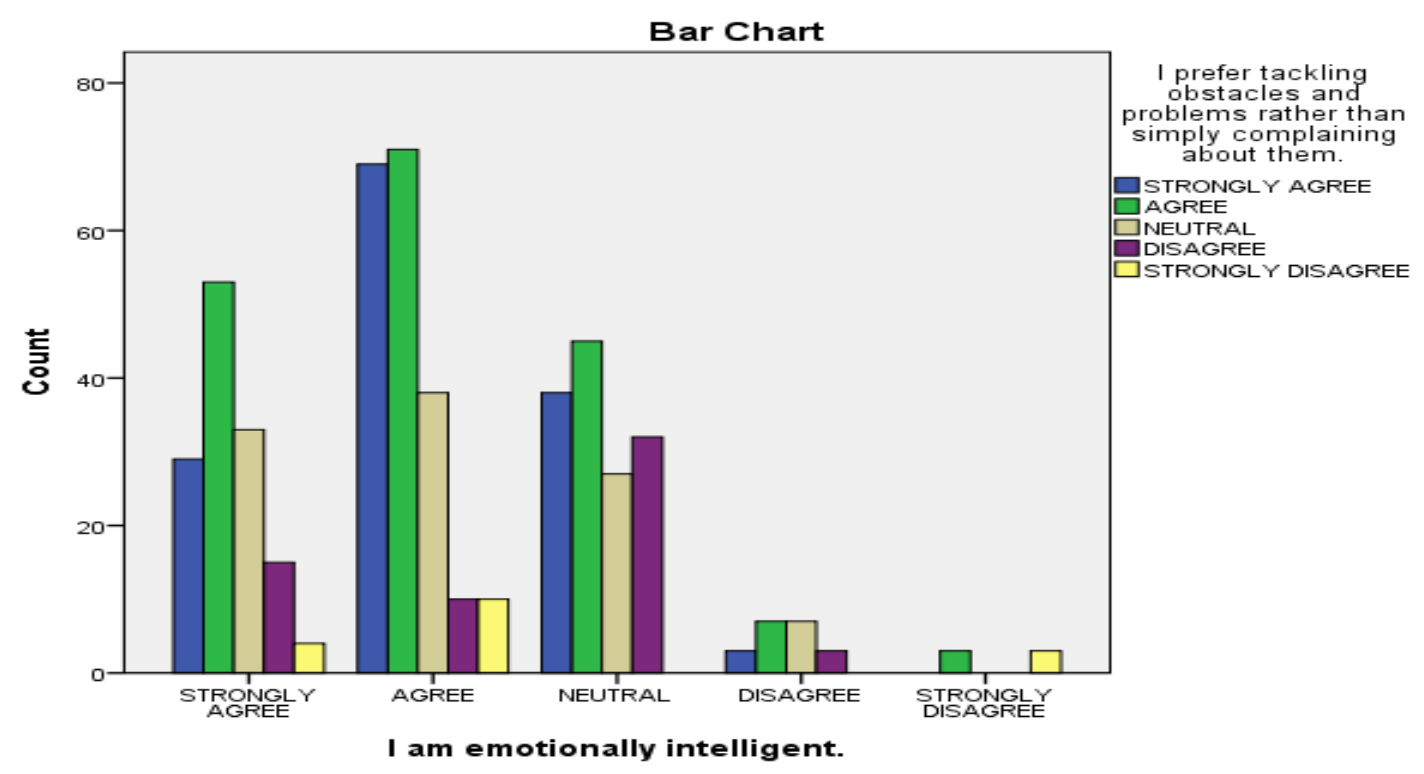

4.2.4 Analysis: Analysis of the relationship between emotional intelligence and'I prefer spotting where personality clashes may impact on work performance' of the software professional in India.

$\mathbf{H}_{0}$ : The two factors are independent.

$\mathbf{H}_{1}$ : The two factors are not independent (associated).

Tool Used: Chi Sauare Test (Analvze $\rightarrow$ Descrintive Statistics $\rightarrow$ Crosstabs)

Table 4.15 Chi-Square Tests

\begin{tabular}{|l|l|l|l|}
\hline Chi-Square Tests & Value & df & Asymp. Sig. (2-sided) \\
\hline & $118.080^{\mathrm{a}}$ & 16 & .000 \\
\hline Pearson Chi-Square & 107.869 & 16 & .000 \\
\hline Likelihood Ratio & 13.078 & 1 & .000 \\
\hline Linear-by-Linear Association & 500 & & \\
\hline N of Valid Cases & r & \\
\hline a. 12 cells (48.0\%) have expected count less than 5. The minimum expected count is .12. \\
\hline
\end{tabular}

Table-4.16. Symmetric Measures:

\begin{tabular}{|c|c|c|c|}
\hline \multicolumn{4}{|l|}{ Symmetric Measures } \\
\hline & & Value & Approx. Sig. \\
\hline Nominal by Nominal & Contingency Coefficient & .437 & .000 \\
\hline \multicolumn{2}{|l|}{$\mathrm{N}$ of Valid Cases } & 500 & \\
\hline \multicolumn{4}{|c|}{ a. Not assuming the null hypothesis. } \\
\hline
\end{tabular}

Interpretation \& Findings: From the table we find out that asymptotic significance for Pearson Chi Square comes out to be 0.000 (less than 0.05 ) so we reject null hypothesis at $5 \%$ level of significance. Hence it can be concluded that two variables are associated. 
Table-4.17. Crosstab: I prefer spotting where personality clashes may impact on work performance.

\begin{tabular}{|c|c|c|c|c|c|c|c|c|}
\hline \multicolumn{9}{|l|}{ Crosstab } \\
\hline & & & \multicolumn{5}{|c|}{$\begin{array}{l}\text { I prefer spotting where personality clashes may impact on work } \\
\text { performance. }\end{array}$} & \multirow[t]{2}{*}{ Total } \\
\hline & & & $\begin{array}{l}\text { STRONGL } \\
\text { Y AGREE }\end{array}$ & AGREE & $\begin{array}{l}\text { NEUTRA } \\
\mathrm{L}\end{array}$ & $\begin{array}{l}\text { DISAGR } \\
\text { EE }\end{array}$ & $\begin{array}{l}\text { STRONGLY } \\
\text { DISAGREE }\end{array}$ & \\
\hline \multirow{10}{*}{$\begin{array}{l}\text { I am emotionally } \\
\text { intelligent. }\end{array}$} & \multirow{2}{*}{$\begin{array}{l}\text { STRONGLY } \\
\text { AGREE }\end{array}$} & Count & 29 & 73 & 21 & 11 & 0 & 134 \\
\hline & & $\%$ of Total & $5.8 \%$ & $14.6 \%$ & $4.2 \%$ & $2.2 \%$ & $0.0 \%$ & $26.8 \%$ \\
\hline & \multirow{2}{*}{ AGREE } & Count & 57 & 114 & 14 & 10 & 3 & 198 \\
\hline & & $\%$ of Total & $11.4 \%$ & $22.8 \%$ & $2.8 \%$ & $2.0 \%$ & $0.6 \%$ & $39.6 \%$ \\
\hline & \multirow{2}{*}{ NEUTRAL } & Count & 14 & 78 & 40 & 3 & 7 & 142 \\
\hline & & $\%$ of Total & $2.8 \%$ & $15.6 \%$ & $8.0 \%$ & $0.6 \%$ & $1.4 \%$ & $28.4 \%$ \\
\hline & \multirow{2}{*}{ DISAGREE } & Count & 10 & 3 & 0 & 7 & 0 & 20 \\
\hline & & $\%$ of Total & $2.0 \%$ & $0.6 \%$ & $0.0 \%$ & $1.4 \%$ & $0.0 \%$ & $4.0 \%$ \\
\hline & \multirow{2}{*}{$\begin{array}{l}\text { STRONGLY } \\
\text { DISAGREE }\end{array}$} & Count & 0 & 0 & 3 & 3 & 0 & 6 \\
\hline & & $\%$ of Total & $0.0 \%$ & $0.0 \%$ & $0.6 \%$ & $0.6 \%$ & $0.0 \%$ & $1.2 \%$ \\
\hline \multirow{2}{*}{\multicolumn{2}{|c|}{ Total }} & Count & 110 & 268 & 78 & 34 & 10 & 500 \\
\hline & & $\%$ of Total & $22.0 \%$ & $53.6 \%$ & $15.6 \%$ & $6.8 \%$ & $2.0 \%$ & $100.0 \%$ \\
\hline
\end{tabular}

Interpretation: from the above crosstab, it can said that out of total 500 respondents (Software Professionals), $22.0 \%$ respondents strongly agreed, 53.6\% respondents agreed, $15.6 \%$ respondents were neutral, $6.8 \%$ respondents disagreed and $2.0 \%$ respondents strongly disagreed that 'I prefer spotting where personality clashes may impact on work performance.

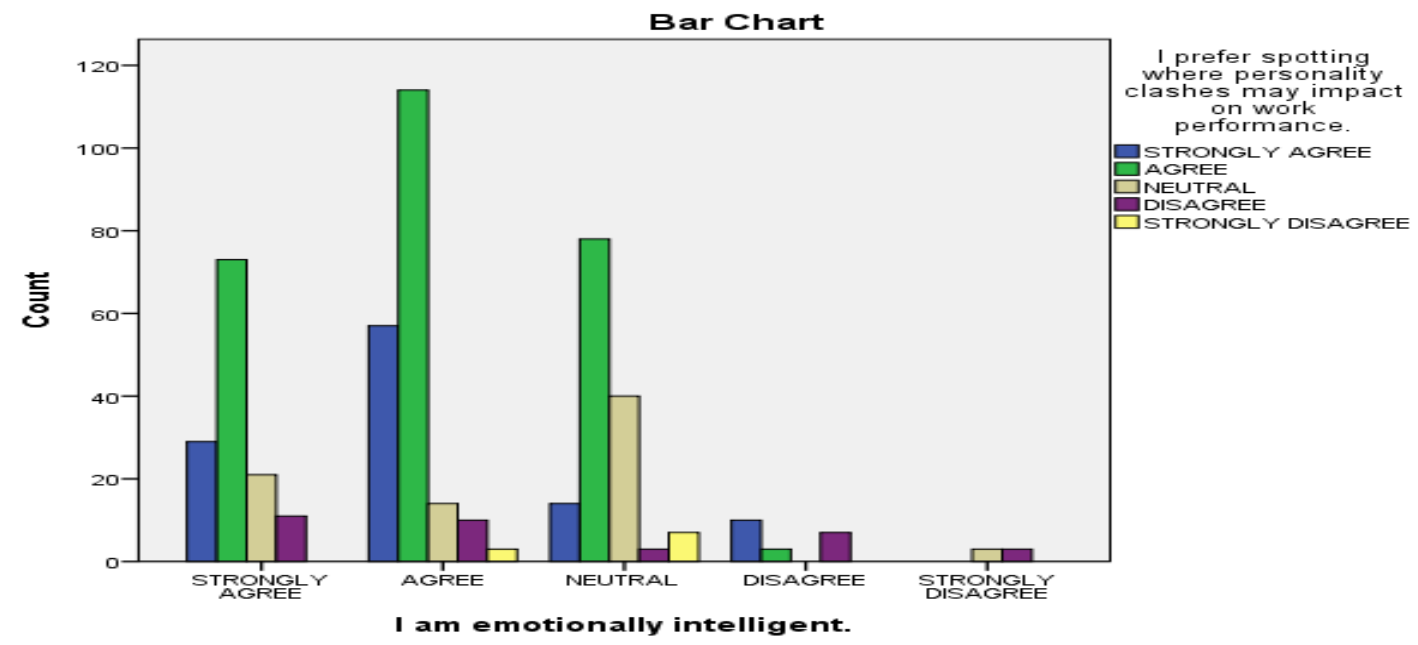

4.2.5 Analysis: Analysis of the relationship between emotional intelligence and' $I$ am able to manage the conflicts and problems faced in my workplace'of the software professional in India.

$\mathbf{H}_{0}$ : The two factors are independent.

$\mathbf{H}_{1}$ : The two factors are not independent (associated).

Tool Used: Chi Square Test (Analyze $\rightarrow$ Descriptive Statistics $\rightarrow$ Crosstabs)

Table 4.18 Chi-Square Tests

\begin{tabular}{|l|l|l|l|}
\hline Chi-Square Tests & Value & df & Asymp. Sig. (2-sided) \\
\hline Pearson Chi-Square & $128.379^{\mathrm{a}}$ & 16 & .000 \\
\hline Likelihood Ratio & 85.841 & 16 & .000 \\
\hline Linear-by-Linear Association & 22.174 & 1 & .000 \\
\hline N of Valid Cases & 500 & & \\
\hline a. 10 cells (40.0\%) have expected count less than 5. The minimum expected count is .16. \\
\hline
\end{tabular}

Table-4.19. Symmetric Measures:

\begin{tabular}{|c|c|c|c|}
\hline \multicolumn{4}{|l|}{ Symmetric Measures } \\
\hline & & Value & Approx. Sig. \\
\hline Nominal by Nominal & Contingency Coefficient & .452 & .000 \\
\hline $\mathrm{N}$ of Valid Cases & & 500 & \\
\hline
\end{tabular}


b. Using the asymptotic standard error assuming the null hypothesis.

Interpretation \& Findings: From the table we find out that asymptotic significance for Pearson Chi Square comes out to be 0.000 (less than 0.05 ) so we reject null hypothesis at $5 \%$ level of significance. Hence it can be concluded that two variables are associated

Table-4.20. Crosstab: I am able to manage the conflicts and problems faced in my workplace.

\begin{tabular}{|c|c|c|c|c|c|c|c|c|}
\hline \multicolumn{9}{|l|}{ Crosstab } \\
\hline & & & \multicolumn{5}{|c|}{$\begin{array}{l}\text { I am able to manage the conflicts and problems faced in my } \\
\text { workplace. }\end{array}$} & \multirow[t]{2}{*}{ Total } \\
\hline & & & $\begin{array}{l}\text { STRONGL } \\
\text { Y AGREE }\end{array}$ & AGREE & $\begin{array}{l}\text { NEUTRA } \\
\text { L }\end{array}$ & $\begin{array}{l}\text { DISAGR } \\
\text { EE }\end{array}$ & $\begin{array}{l}\text { STRONGLY } \\
\text { DISAGREE }\end{array}$ & \\
\hline \multirow{10}{*}{$\begin{array}{l}\text { I am emotionally } \\
\text { intelligent. }\end{array}$} & \multirow{2}{*}{$\begin{array}{l}\text { STRONGLY } \\
\text { AGREE }\end{array}$} & Count & 44 & 51 & 29 & 7 & 3 & 134 \\
\hline & & $\%$ of Total & $8.8 \%$ & $10.2 \%$ & $5.8 \%$ & $1.4 \%$ & $0.6 \%$ & $26.8 \%$ \\
\hline & \multirow{2}{*}{ AGREE } & Count & 67 & 85 & 43 & 3 & 0 & 198 \\
\hline & & $\%$ of Total & $13.4 \%$ & $17.0 \%$ & $8.6 \%$ & $0.6 \%$ & $0.0 \%$ & $39.6 \%$ \\
\hline & \multirow{2}{*}{ NEUTRAL } & Count & 19 & 53 & 49 & 18 & 3 & 142 \\
\hline & & $\%$ of Total & $3.8 \%$ & $10.6 \%$ & $9.8 \%$ & $3.6 \%$ & $0.6 \%$ & $28.4 \%$ \\
\hline & \multirow{2}{*}{ DISAGREE } & Count & 6 & 7 & 3 & 0 & 4 & 20 \\
\hline & & $\%$ of Total & $1.2 \%$ & $1.4 \%$ & $0.6 \%$ & $0.0 \%$ & $0.8 \%$ & $4.0 \%$ \\
\hline & \multirow{2}{*}{$\begin{array}{l}\text { STRONGLY } \\
\text { DISAGREE }\end{array}$} & Count & 3 & 0 & 0 & 0 & 3 & 6 \\
\hline & & $\%$ of Total & $0.6 \%$ & $0.0 \%$ & $0.0 \%$ & $0.0 \%$ & $0.6 \%$ & $1.2 \%$ \\
\hline \multirow{2}{*}{\multicolumn{2}{|c|}{ Total }} & Count & 139 & 196 & 124 & 28 & 13 & 500 \\
\hline & & $\%$ of Total & $27.8 \%$ & $39.2 \%$ & $24.8 \%$ & $5.6 \%$ & $2.6 \%$ & $100.0 \%$ \\
\hline
\end{tabular}

Interpretation \& Findings: from the above crosstab, it can said that out of total 500 respondents (Software Professionals), $27.8 \%$ respondents strongly agreed, $39.2 \%$ respondents agreed, $24.8 \%$ respondents were neutral, $5.6 \%$ respondents disagreed and $2.6 \%$ respondents strongly disagreed that 'I am able to manage the conflicts and problems faced in my workplace.

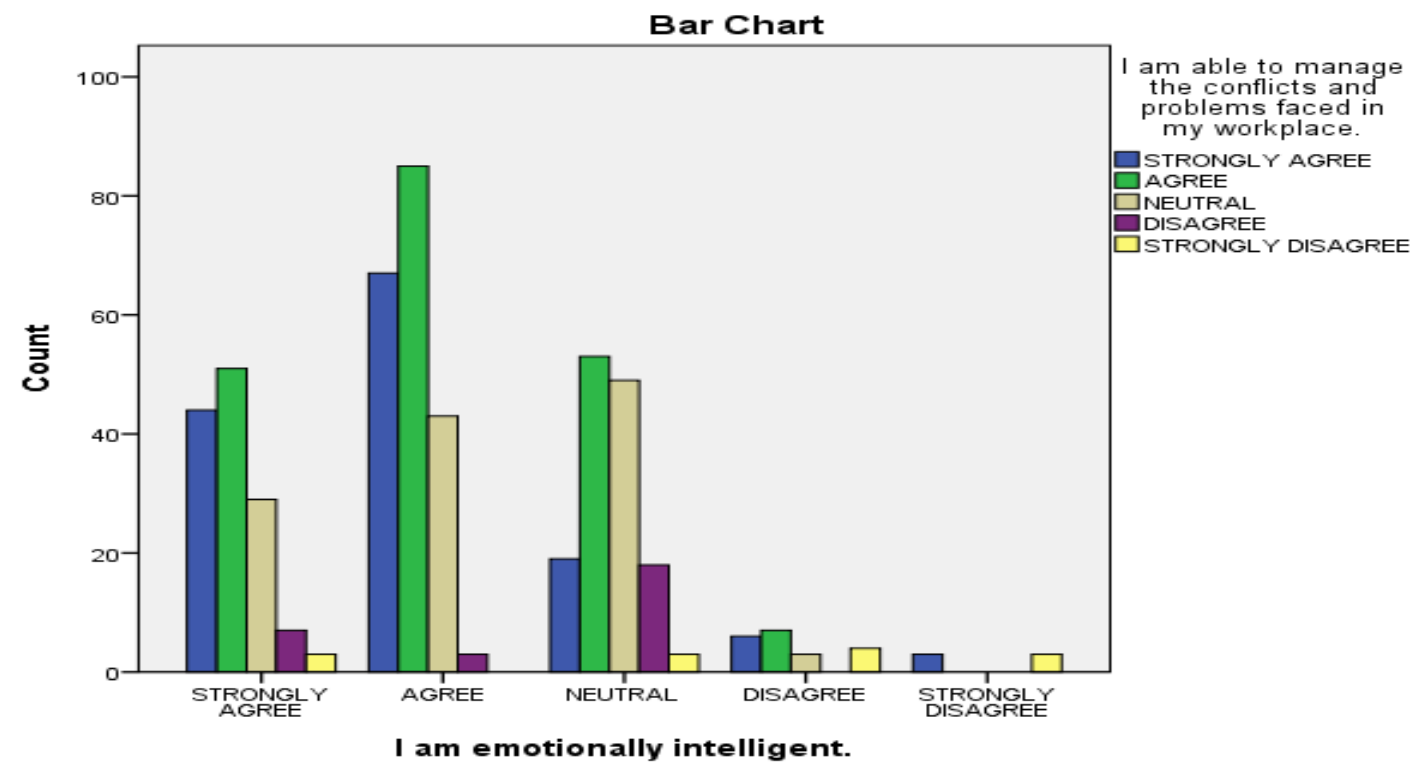

4.2.6 Analysis: Analysis of the relationship between emotional intelligence and' $I$ willingly help others who have work related problems'of the software professional in India.

$\mathbf{H}_{0}$ : The two factors are independent.

$\mathbf{H}_{1}$ : The two factors are not independent (associated).

Tool Used: Chi Sauare Test (Analvze $\rightarrow$ Describtive Statistics $\rightarrow$ Crosstabs)

Table 4.21 Chi-Square Tests

\begin{tabular}{|l|l|l|l|}
\hline Chi-Square Tests & Value & df & Asymp. Sig. (2-sided) \\
\hline & $213.526^{\text {a }}$ & .000 \\
\hline Pearson Chi-Square & 100.299 & 16 & .000 \\
\hline Likelihood Ratio & 50.763 & 16 & .000 \\
\hline Linear-by-Linear Association & 500 & 1 & \\
\hline N of Valid Cases & & \\
\hline a. 12 cells (48.0\%) have expected count less than 5. The minimum expected count is .07. \\
\hline
\end{tabular}


Table-4.22. Symmetric Measures:

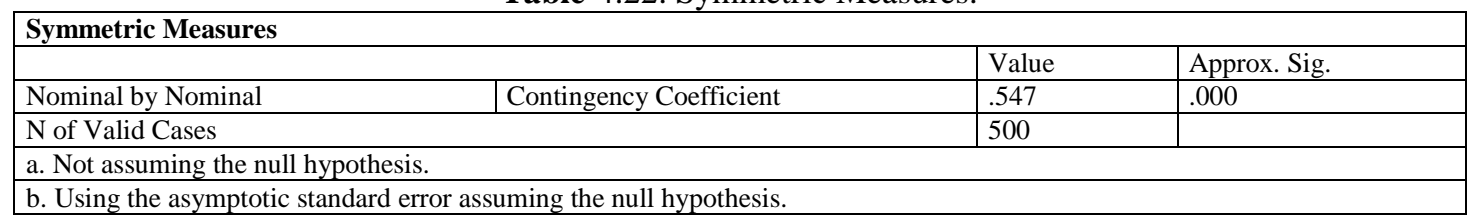

Interpretation \& Findings: From the table we find out that asymptotic significance for Pearson Chi Square comes out to be 0.000 (less than 0.05 ) so we reject null hypothesis at $5 \%$ level of significance. Hence it can be concluded that two variables are associated.

Table-4.23. Crosstab: I willingly help others who have work related problems.

\begin{tabular}{|c|c|c|c|c|c|c|c|c|}
\hline \multicolumn{9}{|l|}{ Crosstab } \\
\hline & & & \multicolumn{5}{|c|}{ I willingly help others who have work related problems. } & \multirow[t]{2}{*}{ Total } \\
\hline & & & $\begin{array}{l}\text { STRONGL } \\
\text { Y AGREE }\end{array}$ & AGREE & $\begin{array}{l}\text { NEUTRA } \\
\mathrm{L}\end{array}$ & $\begin{array}{l}\text { DISAGR } \\
\text { EE }\end{array}$ & $\begin{array}{l}\text { STRONGLY } \\
\text { DISAGREE }\end{array}$ & \\
\hline \multirow{10}{*}{$\begin{array}{l}\text { I am emotionally } \\
\text { intelligent. }\end{array}$} & \multirow{2}{*}{$\begin{array}{l}\text { STRONGLY } \\
\text { AGREE }\end{array}$} & Count & 46 & 56 & 29 & 3 & 0 & 134 \\
\hline & & $\%$ of Total & $9.2 \%$ & $11.2 \%$ & $5.8 \%$ & $0.6 \%$ & $0.0 \%$ & $26.8 \%$ \\
\hline & \multirow{2}{*}{ AGREE } & Count & 39 & 108 & 43 & 8 & 0 & 198 \\
\hline & & $\%$ of Total & $7.8 \%$ & $21.6 \%$ & $8.6 \%$ & $1.6 \%$ & $0.0 \%$ & $39.6 \%$ \\
\hline & \multirow{2}{*}{ NEUTRAL } & Count & 30 & 48 & 41 & 23 & 0 & 142 \\
\hline & & $\%$ of Total & $6.0 \%$ & $9.6 \%$ & $8.2 \%$ & $4.6 \%$ & $0.0 \%$ & $28.4 \%$ \\
\hline & \multirow{2}{*}{ DISAGREE } & Count & 0 & 10 & 3 & 4 & 3 & 20 \\
\hline & & $\%$ of Total & $0.0 \%$ & $2.0 \%$ & $0.6 \%$ & $0.8 \%$ & $0.6 \%$ & $4.0 \%$ \\
\hline & \multirow{2}{*}{$\begin{array}{l}\text { STRONGLY } \\
\text { DISAGREE }\end{array}$} & Count & 0 & 0 & 3 & 0 & 3 & 6 \\
\hline & & $\%$ of Total & $0.0 \%$ & $0.0 \%$ & $0.6 \%$ & $0.0 \%$ & $0.6 \%$ & $1.2 \%$ \\
\hline \multirow{2}{*}{\multicolumn{2}{|c|}{ Total }} & Count & 115 & 222 & 119 & 38 & 6 & 500 \\
\hline & & $\%$ of Total & $23.0 \%$ & $44.4 \%$ & $23.8 \%$ & $7.6 \%$ & $1.2 \%$ & $100.0 \%$ \\
\hline
\end{tabular}

Interpretation \& Findings: from the above crosstab, it can said that out of total 500 respondents (Software Professionals), $23.0 \%$ respondents strongly agreed, $44.4 \%$ respondents agreed, $23.8 \%$ respondents were neutral, $7.6 \%$ respondents disagreed and $1.2 \%$ respondents strongly disagreed that 'I willingly help others who have work related problems.'

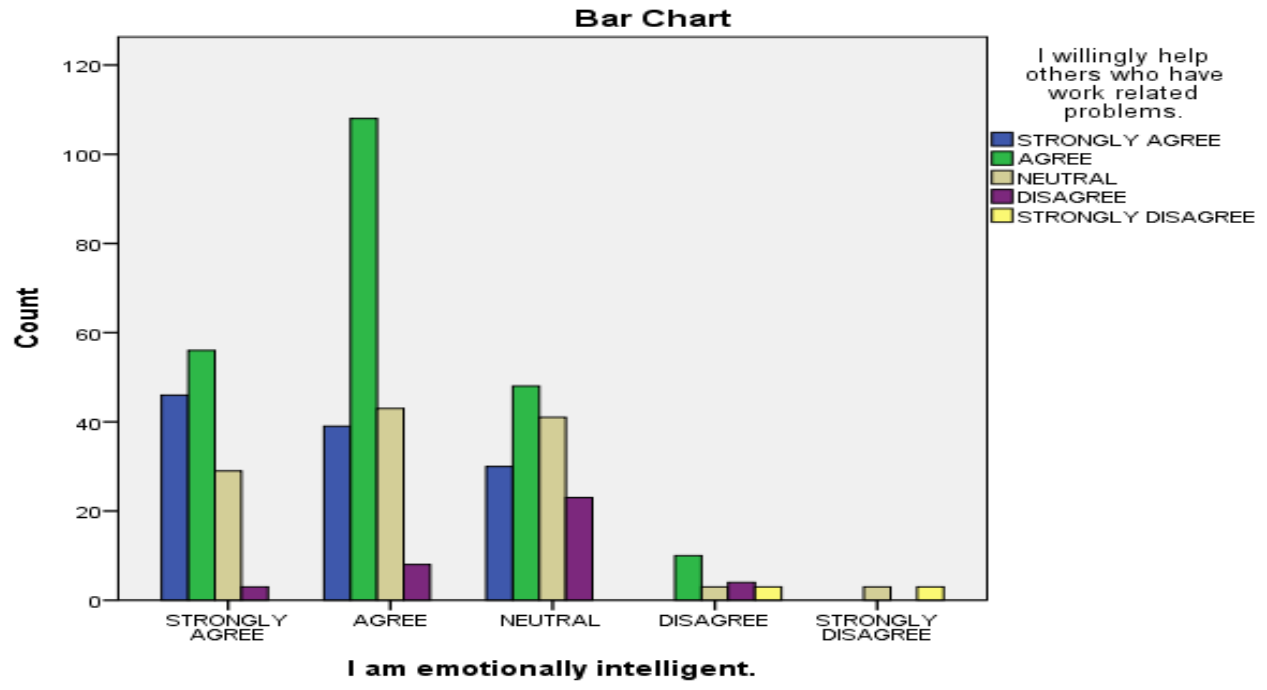

4.2.7 Analysis: Analysis of the relationship between emotional intelligence and'In difficult situations, I put my immediate needs on hold in favour of achieving larger goals' of the software professional in India.

$\mathbf{H}_{0}$ : The two factors are independent.

$\mathbf{H}_{1}$ : The two factors are not independent (associated).

Tool Used: Chi Square Test (Analyze $\rightarrow$ Descriptive Statistics $\rightarrow$ Crosstabs)

Table 4.24 Chi-Square Tests

\begin{tabular}{|l|l|l|l|}
\hline Chi-Square Tests & Value & df & Asymp. Sig. (2-sided) \\
\hline Pearson Chi-Square & $79.596^{\mathrm{a}}$ & 16 & .000 \\
\hline Likelihood Ratio & 67.751 & 16 & .000 \\
\hline Linear-by-Linear Association & 24.018 & 1 & .000 \\
\hline
\end{tabular}


Topic: A study on the association of conflict management competencies with emotional intelligence of

\begin{tabular}{|l|l|l|}
\hline $\mathrm{N}$ of Valid Cases & 500 & \\
\hline a. 11 cells (44.0\%) have expected count less than 5. The minimum expected count is .13. \\
\hline \multicolumn{2}{|c|}{ Table 4.25. Symmetric Measures: } \\
\hline
\end{tabular}

\begin{tabular}{|c|c|c|c|}
\hline \multicolumn{4}{|c|}{ Table-4.25. Symmetric Measures: } \\
\hline \multicolumn{4}{|l|}{ Symmetric Measures } \\
\hline & & Value & Approx. Sig \\
\hline Nominal by Nominal & Contingency Coefficient & .371 & .000 \\
\hline \multicolumn{2}{|l|}{$\mathrm{N}$ of Valid Cases } & 500 & \\
\hline \multicolumn{4}{|c|}{ a. Not assuming the null hypothesis. } \\
\hline
\end{tabular}

Interpretation \& Findings: From the table we find out that asymptotic significance for Pearson Chi Square comes out to be 0.000 (less than 0.05 ) so we reject null hypothesis at $5 \%$ level of significance. Hence it can be concluded that two variables are associated.

Table-4.26. Crosstab: In difficult situations, I put my immediate needs on hold in favour of achieving larger goals.

\begin{tabular}{|c|c|c|c|c|c|c|c|c|}
\hline \multicolumn{9}{|l|}{ Crosstab } \\
\hline & & & \multicolumn{5}{|c|}{$\begin{array}{l}\text { In difficult situations, I put my immediate needs on hold in } \\
\text { favour of achieving larger goals. }\end{array}$} & \multirow[t]{2}{*}{ Total } \\
\hline & & & $\begin{array}{l}\text { STRONG } \\
\text { LY } \\
\text { AGREE }\end{array}$ & AGREE & $\begin{array}{l}\text { NEUTRA } \\
\mathrm{L}\end{array}$ & $\begin{array}{l}\text { DISAGR } \\
\text { EE }\end{array}$ & $\begin{array}{l}\text { STRONGL } \\
\text { Y } \\
\text { DISAGREE }\end{array}$ & \\
\hline \multirow{10}{*}{$\begin{array}{l}\text { I am emotionally } \\
\text { intelligent. }\end{array}$} & \multirow{2}{*}{$\begin{array}{l}\text { STRONGLY } \\
\text { AGREE }\end{array}$} & Count & 32 & 72 & 24 & 6 & 0 & 134 \\
\hline & & $\%$ of Total & $6.4 \%$ & $14.4 \%$ & $4.8 \%$ & $1.2 \%$ & $0.0 \%$ & $26.8 \%$ \\
\hline & \multirow{2}{*}{ AGREE } & Count & 82 & 76 & 29 & 7 & 4 & 198 \\
\hline & & $\%$ of Total & $16.4 \%$ & $15.2 \%$ & $5.8 \%$ & $1.4 \%$ & $0.8 \%$ & $39.6 \%$ \\
\hline & \multirow{2}{*}{ NEUTRAL } & Count & 25 & 67 & 29 & 17 & 4 & 142 \\
\hline & & $\%$ of Total & $5.0 \%$ & $13.4 \%$ & $5.8 \%$ & $3.4 \%$ & $0.8 \%$ & $28.4 \%$ \\
\hline & \multirow{2}{*}{ DISAGREE } & Count & 3 & 4 & 7 & 3 & 3 & 20 \\
\hline & & $\%$ of Total & $0.6 \%$ & $0.8 \%$ & $1.4 \%$ & $0.6 \%$ & $0.6 \%$ & $4.0 \%$ \\
\hline & \multirow{2}{*}{$\begin{array}{l}\text { STRONGLY } \\
\text { DISAGREE }\end{array}$} & Count & 0 & 3 & 0 & 3 & 0 & 6 \\
\hline & & $\%$ of Total & $0.0 \%$ & $0.6 \%$ & $0.0 \%$ & $0.6 \%$ & $0.0 \%$ & $1.2 \%$ \\
\hline \multirow{2}{*}{\multicolumn{2}{|c|}{ Total }} & Count & 142 & 222 & 89 & 36 & 11 & 500 \\
\hline & & $\%$ of Total & $28.4 \%$ & $44.4 \%$ & $17.8 \%$ & $7.2 \%$ & $2.2 \%$ & $100.0 \%$ \\
\hline
\end{tabular}

Interpretation \& Findings: from the above crosstab, it can said that out of total 500 respondents (Software Professionals), $28.4 \%$ respondents strongly agreed, $44.4 \%$ respondents agreed, $17.8 \%$ respondents were neutral, 7.2\% respondents disagreed and $2.2 \%$ respondents strongly disagreed that 'achieving larger goals In difficult situations, I put my immediate needs on..' hold in favour ofachieving larger goals.'

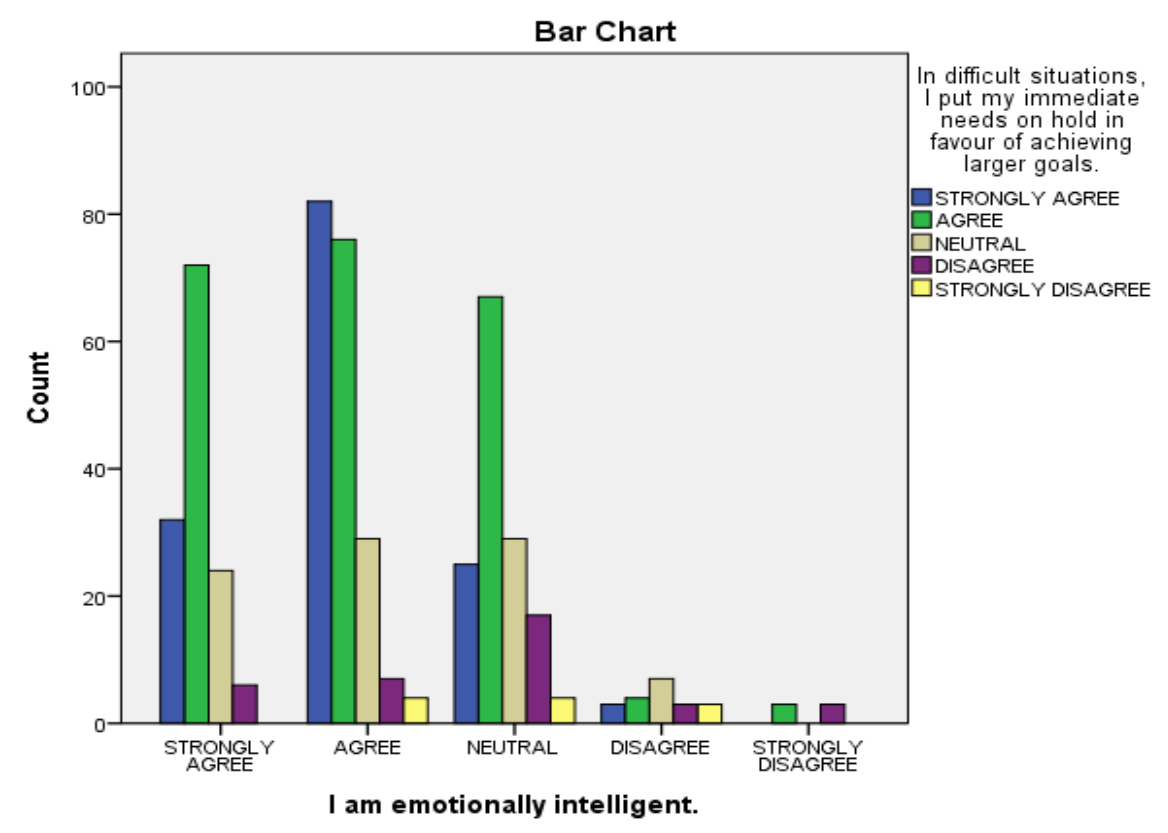

4.2.8 Analysis: Analysis of the relationship between emotional intelligence and' $I$ prefer controlling any potentially emotional outbursts' of the software professional in India.

$\mathbf{H}_{0}$ : The two factors are independent.

$\mathbf{H}_{1}$ : The two factors are not independent (associated).

Tool Used: Chi Sauare Test (Analvze $\rightarrow$ Descrintive Statistics $\rightarrow$ Crosstabs) 
Table 4.27 Chi-Square Tests

\begin{tabular}{|l|l|l|l|}
\hline Chi-Square Tests & Value & df & Asymp. Sig. (2-sided) \\
\hline Pearson Chi-Square & $192.997^{\mathrm{a}}$ & 16 & .000 \\
\hline Likelihood Ratio & 184.527 & 16 & .000 \\
\hline Linear-by-Linear Association & 113.311 & 1 & .000 \\
\hline N of Valid Cases & 500 & & \\
\hline a. 8 cells (32.0\%) have expected count less than 5. The minimum expected count is .29. \\
\hline
\end{tabular}

Table-4.28. Symmetric Measures:

\begin{tabular}{|c|c|c|c|}
\hline \multicolumn{4}{|l|}{ Symmetric Measures } \\
\hline & & Value & Approx. Sig. \\
\hline Nominal by Nominal & Contingency Coefficient & .528 & .000 \\
\hline \multicolumn{2}{|l|}{$\mathrm{N}$ of Valid Cases } & 500 & \\
\hline \multicolumn{4}{|c|}{ a. Not assuming the null hypothesis. } \\
\hline
\end{tabular}

Interpretation \& Findings: From the table we find out that asymptotic significance for Pearson Chi Square comes out to be 0.000 (less than 0.05 ) so we reject null hypothesis at $5 \%$ level of significance. Hence it can be concluded that two variables are associated.

Table-4.29. Crosstab: I prefer controlling any potentially emotional outbursts.

\begin{tabular}{|c|c|c|c|c|c|c|c|c|}
\hline \multicolumn{9}{|l|}{ Crosstab } \\
\hline & & & \multicolumn{5}{|c|}{ I prefer controlling any potentially emotional outbursts. } & \multirow[t]{2}{*}{ Total } \\
\hline & & & $\begin{array}{l}\text { STRONGLY } \\
\text { AGREE }\end{array}$ & AGREE & NEUTRAL & DISAGREE & $\begin{array}{l}\text { STRONGLY } \\
\text { DISAGREE }\end{array}$ & \\
\hline \multirow{10}{*}{$\begin{array}{l}\text { I am emotionally } \\
\text { intelligent. }\end{array}$} & \multirow{2}{*}{$\begin{array}{l}\text { STRONGLY } \\
\text { AGREE }\end{array}$} & Count & 82 & 36 & 7 & 6 & 3 & 134 \\
\hline & & $\%$ of Total & $16.4 \%$ & $7.2 \%$ & $1.4 \%$ & $1.2 \%$ & $0.6 \%$ & $26.8 \%$ \\
\hline & \multirow{2}{*}{ AGREE } & Count & 48 & 95 & 48 & 3 & 4 & 198 \\
\hline & & $\%$ of Total & $9.6 \%$ & $19.0 \%$ & $9.6 \%$ & $0.6 \%$ & $0.8 \%$ & $39.6 \%$ \\
\hline & \multirow{2}{*}{ NEUTRAL } & Count & 10 & 50 & 55 & 17 & 10 & 142 \\
\hline & & $\%$ of Total & $2.0 \%$ & $10.0 \%$ & $11.0 \%$ & $3.4 \%$ & $2.0 \%$ & $28.4 \%$ \\
\hline & \multirow{2}{*}{ DISAGREE } & Count & 0 & 10 & 3 & 3 & 4 & 20 \\
\hline & & $\%$ of Total & $0.0 \%$ & $2.0 \%$ & $0.6 \%$ & $0.6 \%$ & $0.8 \%$ & $4.0 \%$ \\
\hline & \multirow{2}{*}{$\begin{array}{l}\text { STRONGLY } \\
\text { DISAGREE }\end{array}$} & Count & 0 & 0 & 3 & 0 & 3 & 6 \\
\hline & & $\%$ of Total & $0.0 \%$ & $0.0 \%$ & $0.6 \%$ & $0.0 \%$ & $0.6 \%$ & $1.2 \%$ \\
\hline \multirow{2}{*}{\multicolumn{2}{|c|}{ Total }} & Count & 140 & 191 & 116 & 29 & 24 & 500 \\
\hline & & $\%$ of Total & $28.0 \%$ & $38.2 \%$ & $23.2 \%$ & $5.8 \%$ & $4.8 \%$ & $100.0 \%$ \\
\hline
\end{tabular}

Interpretation \& Findings: from the above crosstab, it can said that out of total 500 respondents (Software Professionals), 28.0\% respondents strongly agreed, $38.2 \%$ respondents agreed, $23.2 \%$ respondents were neutral, $5.8 \%$ respondents disagreed and $4.8 \%$ respondents strongly disagreed that 'I prefer controlling any potentially emotional outbursts.'

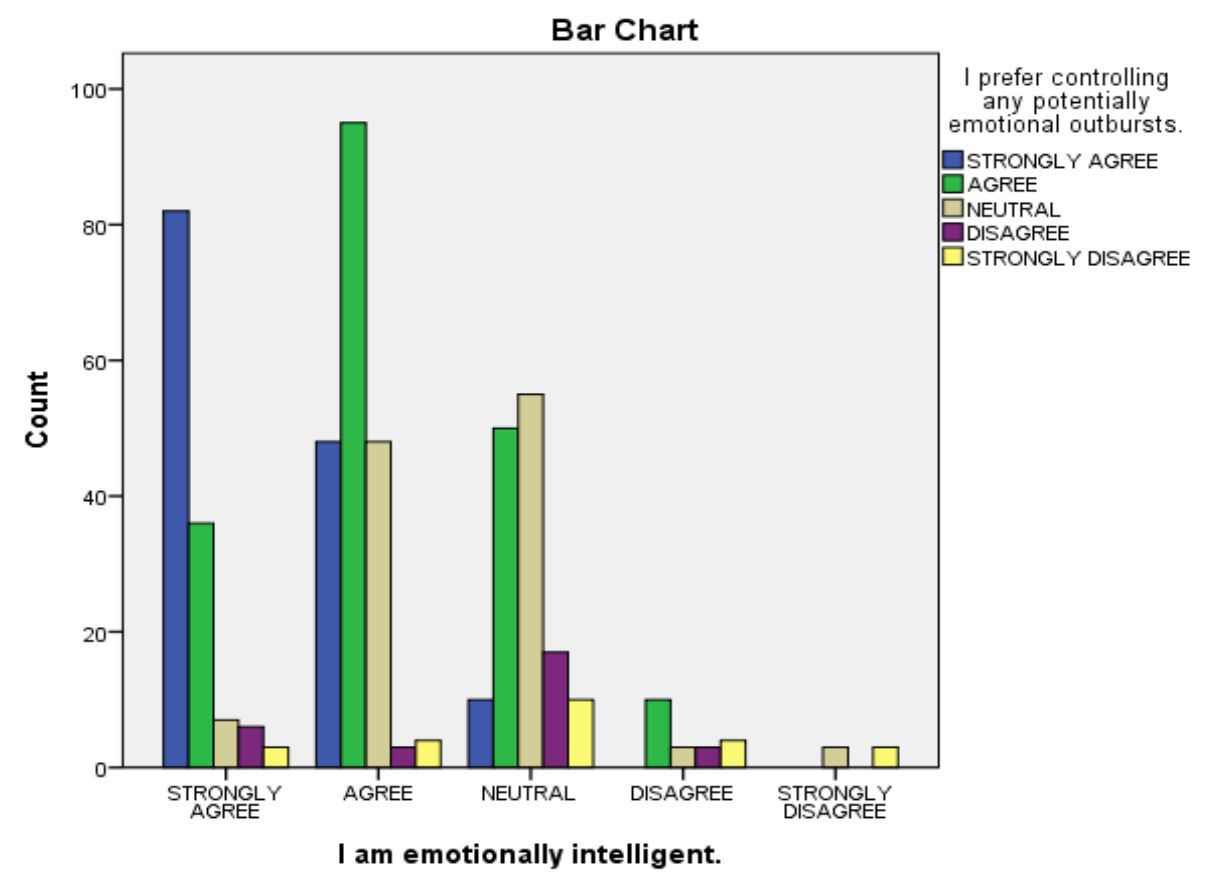


4.2.9 Analysis: Analysis of the relationship between emotional intelligence and 'I prefer holding back from expressing criticism of others'of the software professional in India.

$\mathbf{H}_{0}$ : The two factors are independent.

$\mathbf{H}_{1}$ : The two factors are not independent (associated).

Tool Used: Chi Square Test (Analyze $\rightarrow$ Descriptive Statistics $\rightarrow$ Crosstabs)

Table 4.29 Chi-Square Tests

\begin{tabular}{|l|l|l|l|}
\hline Chi-Square Tests & Value & df & Asymp. Sig. (2-sided) \\
\hline Pearson Chi-Square & $111.314^{\mathrm{a}}$ & 16 & .000 \\
\hline Likelihood Ratio & 119.720 & 16 & .000 \\
\hline Linear-by-Linear Association & 66.376 & 1 & .000 \\
\hline N of Valid Cases & 500 & & \\
\hline a. 10 cells (40.0\%) have expected count less than 5. The minimum expected count is .04. \\
\hline
\end{tabular}

Table-4.30. Symmetric Measures:

\begin{tabular}{|c|c|c|c|}
\hline \multicolumn{4}{|l|}{ Symmetric Measures } \\
\hline & & Value & Approx. Sig. \\
\hline Nominal by Nominal & Contingency Coefficient & .427 & .000 \\
\hline $\mathrm{N}$ of Valid Cases & & 500 & \\
\hline
\end{tabular}

Interpretation \& Findings: From the table we find out that asymptotic significance for Pearson Chi Square comes out to be 0.000 (less than 0.05 ) so we reject null hypothesis at $5 \%$ level of significance. Hence it can be concluded that two variables are associated.

Table-4.31. Crosstab: I prefer holding back from expressing criticism of others.

\begin{tabular}{|c|c|c|c|c|c|c|c|c|}
\hline \multicolumn{9}{|l|}{ Crosstab } \\
\hline & & & \multicolumn{5}{|c|}{ I prefer holding back from expressing criticism of others. } & \multirow[t]{2}{*}{ Total } \\
\hline & & & $\begin{array}{l}\text { STRONGLY } \\
\text { AGREE }\end{array}$ & AGREE & NEUTRAL & DISAGREE & $\begin{array}{l}\text { STRONGLY } \\
\text { DISAGREE }\end{array}$ & \\
\hline \multirow{10}{*}{$\begin{array}{l}\text { I am emotionally } \\
\text { intelligent. }\end{array}$} & \multirow{2}{*}{$\begin{array}{l}\text { STRONGLY } \\
\text { AGREE }\end{array}$} & Count & 57 & 50 & 27 & 0 & 0 & 134 \\
\hline & & $\%$ of Total & $11.4 \%$ & $10.0 \%$ & $5.4 \%$ & $0.0 \%$ & $0.0 \%$ & $26.8 \%$ \\
\hline & \multirow{2}{*}{ AGREE } & Count & 71 & 71 & 47 & 9 & 0 & 198 \\
\hline & & $\%$ of Total & $14.2 \%$ & $14.2 \%$ & $9.4 \%$ & $1.8 \%$ & $0.0 \%$ & $39.6 \%$ \\
\hline & \multirow{2}{*}{ NEUTRAL } & Count & 11 & 51 & 48 & 29 & 3 & 142 \\
\hline & & $\%$ of Total & $2.2 \%$ & $10.2 \%$ & $9.6 \%$ & $5.8 \%$ & $0.6 \%$ & $28.4 \%$ \\
\hline & \multirow{2}{*}{ DISAGREE } & Count & 3 & 7 & 10 & 0 & 0 & 20 \\
\hline & & $\%$ of Total & $0.6 \%$ & $1.4 \%$ & $2.0 \%$ & $0.0 \%$ & $0.0 \%$ & $4.0 \%$ \\
\hline & \multirow{2}{*}{$\begin{array}{l}\text { STRONGLY } \\
\text { DISAGREE }\end{array}$} & \begin{tabular}{|l|} 
Count \\
\end{tabular} & 0 & 3 & 0 & 3 & 0 & 6 \\
\hline & & $\%$ of Total & $0.0 \%$ & $0.6 \%$ & $0.0 \%$ & $0.6 \%$ & $0.0 \%$ & $1.2 \%$ \\
\hline \multirow{2}{*}{\multicolumn{2}{|c|}{ Total }} & \begin{tabular}{|l|} 
Count \\
\end{tabular} & 142 & 182 & 132 & 41 & 3 & 500 \\
\hline & & $\%$ of Total & $28.4 \%$ & $36.4 \%$ & $26.4 \%$ & $8.2 \%$ & $0.6 \%$ & $100.0 \%$ \\
\hline
\end{tabular}

Interpretation \& Findings: from the above crosstab, it can said that out of total 500 respondents (Software Professionals), $28.4 \%$ respondents strongly agreed, $36.4 \%$ respondents agreed, $26.4 \%$ respondents were neutral, $8.2 \%$ respondents disagreed and $0.6 \%$ respondents strongly disagreed that 'I prefer holding back from expressing criticism of others.'

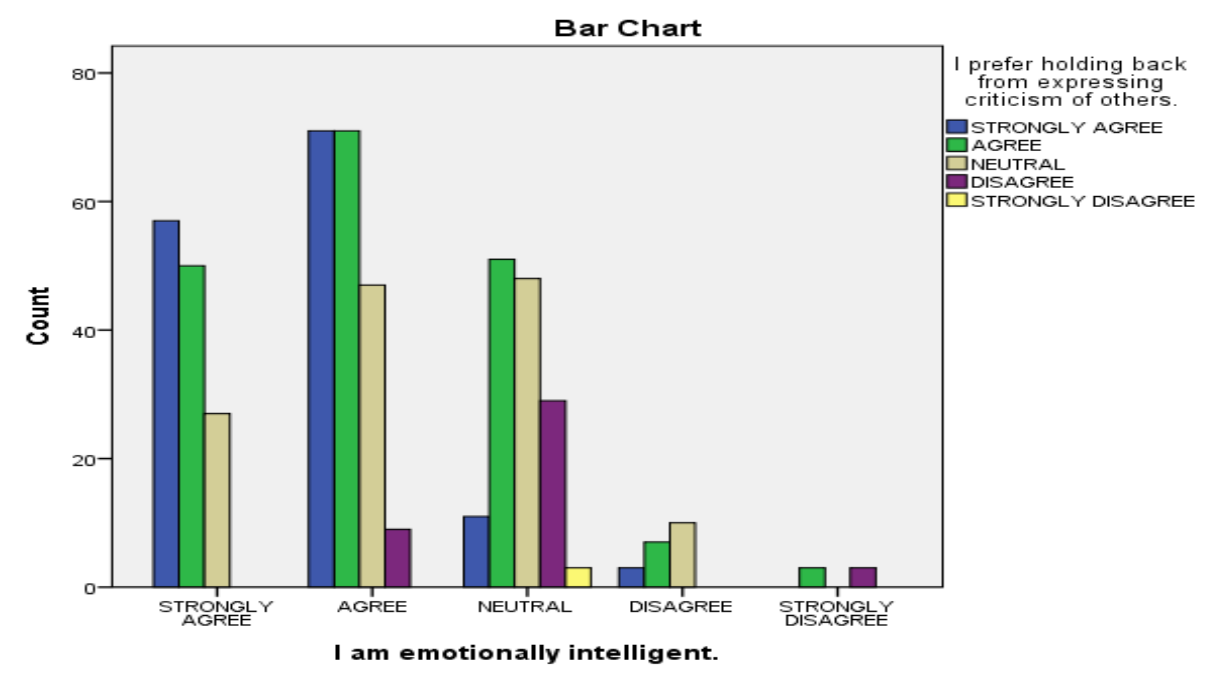




\section{Conclusion, Discussion and Practical Implications}

Software development is a creative and exciting profession where passion and technical knowledgeare not an option but a must competency. Software professionals with a passion for their job are those, who are technically sound, committed, fervent, and, emotionally intelligent in their work as well as with their clients, and are also with their colleagues, family and the society. This optimism is crucial to achieve excellence inthe job. Emotional intelligence is the capability which includesconflict management competencies and skills that influence one's ability to be successful in joband its task even under various pressures.In present study, the association between conflict management competencies and emotional intelligence of software professionals working in IT industry had been checked. The result of the study proves that conflict management competencies are associated with emotional intelligence of software professional. The result is obvious and in compliance with the expectations of researchers.

Following conflict management competencies were identified after validation process during the pilot study and were finalized to test their association with EI of software professionals-

- I bring disagreements into the open in order to de-escalate it.

- I carefully arrange win-win solutions.

- I prefer tackling obstacles and problems rather than simply complaining about them.

- I prefer spotting where personality clashes may impact on work performance.

- I am able to manage the conflicts and problems faced in my workplace.

- I willingly help others who have work related problems.

- In difficult situations, I put my immediate needs on hold in favour of achieving larger goals.

- I prefer controlling any potentially emotional outbursts.

- I prefer holding back from expressing criticism of others.

Emotional intelligence is essential for the development of conflict management competencies. Both are important and conflict management competencies are theend product of emotional intelligence. If proper conflict management competenciesare not developed, there can be conflicts and misunderstandingsduring the service delivery process while expressing one's emotions or understanding clients' / other's emotions.

Software professionals must learn to bring disagreements into the open in order to de-escalate it and to hold them back from expressing criticism of clients or others. They ought to empathise with clients and make them understand their point of view also and carefully arrange win-win solutions for both the stakeholders. EI of Software professionals' approach must be to prefer tackling obstacles and problems rather than simply complaining about them. They will have to realise that they have to satisfy the clients and solve the issues and conflicts. If they will complain then who will provide solutions to the problems of clients. They must be able to manage the conflicts and problems faced in my workplace and willingly, help others who have work related problems. Software professionals should show empathise with their clients and they can also spot where personality clashes may impact on work performance. In difficult situations, they should put their immediate needs on hold in favour of achieving larger goals or satisfying and serving clients. EI certainly helps them to control any potentially emotional outbursts that may have dire consequences and may affect performance adversely.

Conflict management incompetencewill certainly affect individual performance as well as organizational performance adversely. Emotionally intelligent Software professionals have anobligation to their clients, to accept responsibility and accountability. They are well aware, empathetic and compassionate in their professional relationships with their client and everyone in the organization.

\section{References and Bibliography}

[1] Birks, Y. F., \& Watt, I. S. (2007). Emotional intelligence and patient-centred care. Journal of the Royal Society of Medicine, $100(8), 368-374$.

[2] Boyatzis,R.E., andRatti,F.(2009).“Emotional,SocialandCognitiveIntelligence Competencies Distinguishing Effective Italian Managers and Leaders in a Private Companyand Cooperatives", Journal of Management Development, Vol 28 (9), 821-838.

[3] Carmeli, A. (2003).“The Relationship betweenEmotional Intelligence and Work Attitudes,BehaviorandOutcomes",JournalofManagerialPsychology,Vol 18, pp 788-813.

[4] Gardner, H. (1983). Frames of mind: The theory of multiple intelligences. New York: Basic Books

[5] Gardner, H. (1995). Cracking open the IQbox. In S. Fraser (Ed.), The bell curve wars (pp.23-35). New York: Basic Books.

[6] Goleman, D. (1995). Emotional intelligence: Why it can matter more than IQ. New York, NY: Bantam.

[7] Goleman, D., Boyatzis, R., \& McKee, R. (2002). Primal leadership: Realizing the power of emotional intelligence. Boston, MA: Harvard Business School Press.

[8] Salovey, P. and Mayer, J.D. (1990). Emotional Intelligence. Imagination, Cognition and Personality. 9, 185-211Goleman, D., McKee, A., \&Boyayzis, R. (2006). Primal leadership: Realizing the power of emotional intelligence. Nursing News, 30(3), 24-24.

[9] Salovey, P., Bedell, B. T., Detweiller, J. B., \& Mayer, J. D. (2000). Coping intelligently: Emotional intelligence and the coping process. In C. R. Snyder (Ed.), Coping: The psychology of what works (pp. 141-164). New York: Oxford University Press.

[10] Salovey, P., Stroud, L. R., Woolery, A., \&Epel, E. S. (2002). Perceived emotional intelligence, stress reactivity, and symptom reports: Further explorations using the trait meta-mood scale. Psychology \& Health, 17, 611-627. 
[11] Salovey, P., Mayer, J. D., Goldman, S. L., Turvey, C., \&Palfai, T. P. (1995). Emotional attention, clarity, and repair: Exploring emotional intelligence using the trait meta-mood scale. In J. W. Pennebaker (Ed.), Emotion, disclosure, \& health (pp. 125-154), Washington, DC: American Psychological Association.

[12] Sternberg, R. J. (1997).Thinking styles.New York: Cambridge University Press.

[13] Sternberg, R. J. (1989). The triarchic mind: A new theory of human intelligence. New York: Penguin Books.

[14] Sternberg, R. J. (1994). Commentary: Reforming school reform: Comments on "multiple intelligences: The theory in practice.". Teachers College Record, 95(4), 561-69.

[15] Sternberg, R. J. (2000). Handbook of intelligence Cambridge; New York: Cambridge University Press, 2000.

[16] Thorndike,E.L.(1920).Intelligenceanditsuse.Harper'sMagazine, 140,227-235. 